\title{
Difference of walking plantar loadings in experienced and novice long-distance runners
}

\author{
ZIXIANG GAO ${ }^{1,2}$, QICHANG MeI ${ }^{1,2,3}$, LiAnGLIANG XIANG ${ }^{1,2}$, YAODONG GU ${ }^{1,2,3 *}$ \\ ${ }^{1}$ Faculty of Sports Science, Ningbo University, Ningbo, China. \\ ${ }^{2}$ Research Academy of Grand Health, Ningbo University, Ningbo, China. \\ ${ }^{3}$ Auckland Bioengineering Institute, University of Auckland, Auckland, New Zealand.
}

\begin{abstract}
Purpose: Long-distance running has been a global popularity, and differences may be observed in runners of experience. This study was aimed to evaluate the differences in plantar loading between experienced long-distance runners and novice runners during walking. The hypotheses were that smaller load in the middle of the forefoot and the stable foot balance would be observed in experienced runners during walking. Methods: Twenty-five participants were recruited as the experienced runners group (ten: frequency $\geq 4$ times/week and distance $\geq 40 \mathrm{~km} /$ week, for over 1 year) and the novice group (fifteen: frequency $\leq 2$ times/week and distance $\leq 10 \mathrm{~km} /$ week, for less than 1 year). Results: The peak Meta loading showed that the novice runners were significantly larger than the experienced runners $(p \leq .001)$. The occurring time of peak Meta loading was significantly delayed compared to the experienced runners $(p=.050)$. Peak Foot balance was higher in the novice runners group $(p \leq 0.001)$. Conclusions: This study highlighted the findings of the potential differences of plantar pressures and foot functional parameters during walking in experienced runners and novice runners, specifically the contact duration of phases during stance, toes functions and medial-lateral loading shifting (foot balance) and metatarsals (central forefoot) offloading.
\end{abstract}

Key words: prolonged running, novice, experienced, plantar pressure, statistical parametric mapping

\section{Introduction}

Over the past decades, the popularity of long-distance running has grown around the globe. This could be reflected by the significant incrementing numbers of amateur and professional runners around the world [13]. Functional adaptation is a phenotypic feature in biology, which is relative to selection demands of the external environment [3], causing adaptive changes in motor system, such as the previously reported functions in the toes [16]. One key to improve athletic performance is to make the motor system continuously evolve through the external stimulation, thus leading to adaptation from physiological support. Experienced longdistance runners presented adaptation in cardiovascular and musculoskeletal systems from long-term enduring run compared to the sedentary populations [10].
Though the benefits of long-distance running in health, it may increase the injury risks in runners caused by the accumulative impact from continuous and repetitive running gaits, particularly loadings to joints in lower extremity [17]. Additional evidence indicated that prolonged running participants accounted for up to $52 \%$ of all running-related injuries [12]. Therefore, pains and injuries to the muscles and bones of the lower limb from prolonged running have been focused in recent years [21], [26]. Previous study found that novice runners have a higher risk of running-related injuries, which was due to change of plantar pressure and foot roll-over deviations from running-induced fatigued [2].

Studies on the lower limb and foot biomechanics reported that the plantar pressure measurements could collect useful parameters and metrics to investigate foot functions [8], [23]. Understanding the distribu-

\footnotetext{
* Corresponding author: Yaodong Gu, Faculty of Sports Science, Ningbo University, No. 818, Fenghua Road, Jiangbei District, Ningbo, Zhejiang, China, 315211. E-mail: guyaodong@hotmail.com

Received: April 16th, 2020

Accepted for publication: June 9th, 2020
} 
tions of plantar pressure is of great significance for gait research, footwear design, and potential injury prevention [20], [23]. Running footwear of different functional properties were prescribed to prevent runningrelated injuries [20]. A report indicated that a single long-distance run would increase the loading in the metatarsal bones, thus increasing the risk of stress fracture in the metatarsal bones [21]. Hawrylak et al. [6] revealed that runners involved in prolonged running activities showed greater lateral plantar pressure in gait than untrained individuals. However, whether these differences exist between experienced long-distance runners and novice runners is unknown.

Several previous studies have reported the differences of plantar pressure before and after a single marathon on running plantar pressure [18]. However, to our knowledge, no existing studies have investigated the differences of walking plantar pressures and foot functional parameters in experienced longdistance runners and novice runners, which are also quite crucial for the understanding of adaptation and potential injury risks. In the current study, the walking plantar pressures of experienced long-distance runners and physical active novice runners were collected and analyzed. It was to evaluate the difference in the foot loadings and functions between experienced long-distance runners and novice runners. The proposed hypotheses were that (1) the experienced group would present smaller loadings in the middle of the forefoot than the novice group; (2) the experienced group would exhibit a more stable foot balance during walking.

\section{Methods}

\section{Participants}

Twenty-five participants took part in this study, whom were classified as the experienced runners and novice runners group, were as per recently established criteria [11]. The ten experienced long-distance runners (age: $25 \pm 2.80$ years, height: $1.65 \pm 0.47 \mathrm{~m}$, mass: $66 \pm 10.70 \mathrm{~kg}$, BMI: $23.82 \pm 0.95 \mathrm{~kg} / \mathrm{m}^{2}$ ) all had a history of half or full marathon running and with an average 4 times/week frequency and running distance of $40 \mathrm{~km} /$ week during the past two years. The control group were fifteen physically active novice runners (age: $24 \pm 3.30$ years, height: $1.73 \pm 0.50 \mathrm{~m}$, mass: $68.7 \pm 10.10 \mathrm{~kg}$, BMI: $22.93 \pm 1.82 \mathrm{~kg} / \mathrm{m}^{2}$ ), who started running within one year and ran less than 2 times/week (with running time less than 45 min or total distance less than $10 \mathrm{~km}$ ). All participants had no foot deformities, injuries or surgery to the lower limb in the previous year, and other factors affecting the gait patterns. This study was approved by the Ethics Committee of in the University. Before the experiments, all participants were given informed consent, knowing the objectives, procedures and requirements of the test.

\section{Test protocol}

The walking plantar pressure was recorded using a Footscan ${ }^{\circledR}$ plantar pressure plate at $480 \mathrm{~Hz}$ (RsScan International, $2 \mathrm{~m} \times 0.4 \mathrm{~m} \times 0.02 \mathrm{~m}$, with 16,384 sensors, Olen, Belgium). The plate was mounted on the ground at the center of a 20-meter walkway. Prior to the test, participants were instructed to walk barefoot on the walkway for warm-up and lab familiarization, including walking step adjustment. The walkway was covered with a 3-mm thick rubber mat to form an even surface to stimulate natural gait environment. All participants walked on the walkway with a self-selected speed, which was recorded to be around the speed of $6 \mathrm{~km} / \mathrm{h}$. Five valid trials were included, and a trial was considered valid as following criteria was met: (1) two consecutive steps on the plate, (2) no adjustment of step while crossing the plate, and (3) presence of a heel-strike walking pattern [5].

The plantar pressure was divided into ten anatomical regions using the RsScan software system, including hallux $(\mathrm{H})$, other toes (OT), metatarsal I-V (M1-M5), mid foot (MF), medial heel (MH), and lateral heel $(\mathrm{LH})$. For the accuracy of the data, all regions of interest were automatically divided by the system, then manually checked and adjusted by an expert foot biomechanics researcher. The stance was separated into four phases, including initial contact phase (ICP), forefoot contact phase (FFCP), foot flat phase (FFP) and forefoot push off phase (FFPOP), as per previously established protocol [15].

\section{Data processing and statistical analysis}

The parameters, including maximal force, peak pressure and impulse, in plantar pressure analysis were normalized using the total force (Zavg) in this study following a previously published method [28]. This normalization was proven to eliminate influence from participants' individual body weight and walking speed [28]. Zavg was computed using the sum of force divided by the sum of data frames. The contact area of each region was normalized by the total foot contact area to avoid foot size effect. This study also employed temporal indices/ratio calculations (T-IRT) as 
a functional interpretation of foot pressure measurement. The parameters of Foot balance (1) and Meta loading (2) were used as indicators to assess the medial and lateral loading (foot balance) in the foot and force distribution in central forefoot (M2 and M3) during stance [28].

$$
\begin{gathered}
\text { Foot balance }=[(\mathrm{MH}+\mathrm{M} 1+\mathrm{M} 2) \\
-(\mathrm{LH}+\mathrm{M} 4+\mathrm{M} 5)] \times 100 / \text { Zavg }
\end{gathered}
$$

$$
\text { Meta loading }=[(\mathrm{M} 2+\mathrm{M} 3)
$$

$$
-(\mathrm{M} 1+\mathrm{M} 4+\mathrm{M} 5)] \times 100 / \mathrm{Zavg}
$$

The maximal force, contact area, peak pressure, impulse, time-series of force, time of peak force, contact duration, and time-series of functional parameters were taken for statistical analysis. Data normality was checked prior to the statistical analysis using the Shapiro-Wilk test. The independent-sample $t$-test was employed to check the differences between the experienced runners and novice runners. The SPSS 17.0 software (SPSS Inc., Chicago, IL, USA) was employed to check the significance of plantar pressure parameters, including maximal force, contact area, peak pressure, impulse and contact duration. Due to the 1-dimensional time-varying characteristics of force and function parameters, the independentsample $t$-test package in the open source Statistical Parametric Mapping 1d (SPM1d), depending on random vector field theory to check data variability [22], was used for statistical analysis in the MATLAB
R2018a (The MathWorks, MA, USA). The significance level was set at 0.05 .

\section{Results}

\section{Contact area and contact duration}

Contact area was normalized by the total area in percentage. Contact area at $\mathrm{H}$ of the experienced runners was significantly $(p=0.040)$ smaller than the novice group, but M1 was greater $(p=0.040)$ than the control group. Contact duration presented significantly shorter time in the ICP $(p=0.220), \operatorname{FFCP}(p=0.030)$, and FFPOP $(p=0.040)$ of the experienced runners group (Table 1). No difference was observed in FFP.

\section{Plantar pressure distribution}

The plantar pressure data, including maximum force, force time integral (impulse) and peak pressure were normalized by Zavg in percentage. As in Table 2, the peak pressure $(p=0.040)$ and impulse $(p=0.043)$ in OT of the experienced runners were significantly higher than the novice group. The peak pressure ( $p=$ 0.042 ) in $\mathrm{M} 2$ of the experienced runners was smaller than the novice group. The maximal force $(p=0.028)$ and peak pressure $(p=0.018)$ in M3 of the experienced runners were smaller than the novice group. The maximal force $(p \leq 0.001)$, peak pressure $(p \leq$

\begin{tabular}{|c|c|c|c|c|c|}
\hline & Marathon group & Control group & $p$-value & Confidence interval (95\%) & Effect size \\
\hline \multicolumn{6}{|l|}{ Contact area } \\
\hline $\mathrm{H}$ & $8.29(1.34)$ & $9.24(0.85)$ & $0.04 *$ & $(0.02 \sim 1.87)$ & 0.39 \\
\hline OT & $7.63(2.13)$ & $8.09(1.96)$ & 0.59 & $(-1.27 \sim 2.20)$ & 0.11 \\
\hline M1 & $10.71(0.67)$ & $9.68(1.40)$ & $0.04 *$ & $(-2.01 \sim 0.60)$ & 0.43 \\
\hline M2 & $7.77(0.29)$ & $8.63(2.03)$ & 0.19 & $(-0.47 \sim 2.18)$ & 0.28 \\
\hline M3 & $6.26(0.45)$ & $7.01(1.57)$ & 0.15 & $(-0.29 \sim 1.80)$ & 0.31 \\
\hline M4 & $6.38(0.44)$ & $6.84(0.80)$ & 0.11 & $(-0.11 \sim 1.04)$ & 0.34 \\
\hline M5 & $6.02(0.83)$ & $5.69(0.67)$ & 0.29 & $(-0.96 \sim 0.30)$ & 0.21 \\
\hline MF & $23.45(3.58)$ & $20.97(5.00)$ & 0.19 & $(-6.28 \sim 1.30)$ & 0.26 \\
\hline $\mathrm{MH}$ & $12.58(0.75)$ & $12.93(1.34)$ & 0.47 & $(-0.62 \sim 1.30)$ & 0.16 \\
\hline LH & $10.91(0.74)$ & $10.93(0.80)$ & 0.94 & $(-0.64 \sim 0.68)$ & 0.01 \\
\hline \multicolumn{6}{|c|}{ Contact duration } \\
\hline ICP & $39.65(11.62)$ & $56.10(19.02)$ & $0.22 *$ & $(2.54 \sim 30.35)$ & 0.46 \\
\hline FFCP & $44.65(19.53)$ & $83.12(54.60)$ & 0.03* & $(5.26 \sim 71.67)$ & 0.42 \\
\hline FFP & $264.00(66.71)$ & $225.5(71.65)$ & 0.77 & $(-67.83 \sim 50.87)$ & 0.06 \\
\hline FFPOP & $264.82(73.95)$ & $301.8(44.69)$ & $0.04 *$ & $(1.23 \sim 72.80)$ & 0.35 \\
\hline
\end{tabular}
$0.001)$ and impulse $(p \leq 0.001)$ in $\mathrm{MH}$ of the experienced runners were significantly decreased.

Table 1. Comparisons of contact area and contact duration in the Marathon and Control groups

Note: “*” means significant difference between the two groups $(p \leq 0.05)$. 
Table 2. Maximal force/Zavg $\times 100$, Peak pressure/Zavg $\times 100$, and Force time integral/Zavg $\times 100$ (Zavg $=$ total number of force / total number of data frames)

\begin{tabular}{|c|c|c|c|c|c|}
\hline & Marathon group & Control group & $p$-value & Confidence interval (95\%) & Effect size \\
\hline \multicolumn{6}{|l|}{ Maximal force } \\
\hline $\mathrm{H}$ & $17.54(6.97)$ & $19.56(8.73)$ & 0.55 & $(-4.83 \sim 8.87)$ & 0.13 \\
\hline OT & $6.98(3.96)$ & $3.95(1.70)$ & $0.04 *$ & $(-5.91 \sim-1.45)$ & 0.45 \\
\hline M1 & $27.84(7.75)$ & $23.37(6.05)$ & 0.13 & $(-10.29 \sim 1.34)$ & 0.31 \\
\hline M2 & $42.76(6.17)$ & $48.90(9.38)$ & 0.08 & $(-0.83 \sim 13.11)$ & 0.36 \\
\hline M3 & $34.23(5.93)$ & $39.16(4.40)$ & $0.03 *$ & $(0.58 \sim 9.28)$ & 0.42 \\
\hline M4 & $24.76(7.69)$ & $20.84(5.50)$ & 0.16 & $(-9.48 \sim 1.64)$ & 0.28 \\
\hline M5 & $11.21(5.20)$ & $8.22(2.75)$ & 0.12 & $(-6.86 \sim 0.86)$ & 0.34 \\
\hline MF & $29.04(12.17)$ & $21.13(11.85)$ & 0.12 & $(-18.13 \sim 2.31)$ & 0.31 \\
\hline $\mathrm{MH}$ & $42.87(7.25)$ & $58.80(11.68)$ & $0.00 * *$ & $(7.36 \sim 24.50)$ & 0.63 \\
\hline LH & $42.56(10.63)$ & $43.35(10.02)$ & 0.86 & $(-7.99 \sim 9.56)$ & 0.04 \\
\hline \multicolumn{6}{|l|}{ Peak pressure } \\
\hline $\mathrm{H}$ & $1.34(0.52)$ & $1.44(0.64)$ & 0.68 & $(-0.40 \sim 0.60)$ & 0.09 \\
\hline OT & $0.54(0.28)$ & $0.36(0.22)$ & 0.10 & $(-0.39 \sim 0.03)$ & 0.33 \\
\hline M1 & $1.62(0.43)$ & $1.58(0.37)$ & 0.84 & $(-0.37 \sim 0.30)$ & 0.04 \\
\hline M2 & $3.33(0.74)$ & $4.20(1.12)$ & $0.04 *$ & $(0.04 \sim 1.70)$ & 0.42 \\
\hline M3 & $3.41(0.67)$ & $4.10(0.65)$ & 0.02 & $(0.13 \sim 1.25)$ & 0.46 \\
\hline M4 & $2.46(0.75)$ & $2.12(0.48)$ & 0.19 & $(-0.86 \sim 0.18)$ & 0.26 \\
\hline M5 & $1.12(0.58)$ & $0.98(0.26)$ & 0.45 & $(-0.58 \sim 0.27)$ & 0.17 \\
\hline MF & $0.83(0.48)$ & $0.63(0.29)$ & 0.21 & $(-0.53 \sim 0.12)$ & 0.25 \\
\hline $\mathrm{MH}$ & $2.09(0.49)$ & $3.24(0.86)$ & $0.00 * *$ & $(0.54 \sim 1.78)$ & 0.64 \\
\hline LH & $2.39(0.80)$ & $2.77(0.72)$ & 0.24 & $(-0.27 \sim 1.03)$ & 0.24 \\
\hline Meta loading & $37.00(11.81)$ & $225.07(122.67)$ & $0.00 * *$ & $(117.47 \sim 258.68)$ & 0.73 \\
\hline Foot Balance & $17.73(20.57)$ & $137.08(76.73)$ & $0.00 * *$ & $(73.90 \sim 167.79)$ & 0.72 \\
\hline \multicolumn{6}{|l|}{ Impulses } \\
\hline $\begin{array}{r}\mathrm{H} \\
\end{array}$ & $2.86(1.10)$ & $3.62(2.35)$ & 0.34 & $(-0.87 \sim 2.40)$ & 0.20 \\
\hline OT & $1.05(0.56)$ & $0.64(0.37)$ & $0.04 *$ & $(-0.80 \sim 0.01)$ & 0.39 \\
\hline M1 & $5.85(1.38)$ & $5.29(1.30)$ & 0.32 & $(-1.69 \sim 0.58)$ & 0.20 \\
\hline M2 & $10.94(2.34)$ & $12.87(3.12)$ & 0.11 & $(-0.47 \sim 4.33)$ & 0.33 \\
\hline M3 & $9.42(1.96)$ & $10.90(2.46)$ & 0.12 & $(-0.44 \sim 3.42)$ & 0.32 \\
\hline M4 & $7.20(2.55)$ & $6.16(1.85)$ & 0.26 & $(-2.89 \sim 0.83)$ & 0.23 \\
\hline M5 & $2.93(1.42)$ & $2.17(0.87)$ & 0.12 & $(-1.73 \sim 0.20)$ & 0.31 \\
\hline MF & $6.26(3.41)$ & $5.36(3.65)$ & 0.55 & $(-3.93 \sim 2.13)$ & 0.13 \\
\hline MH & $7.87(1.93)$ & $12.51(4.56)$ & $0.01 * *$ & $(1.50 \sim 7.79)$ & 0.55 \\
\hline LH & $7.47(2.07)$ & $9.14(3.00)$ & 0.14 & $(-0.59 \sim 3.93)$ & 0.31 \\
\hline
\end{tabular}

Note: “*” means significant difference between the two groups $(p \leq 0.05)$; “**” means significant difference between the two groups $(p \leq 0.01)$.
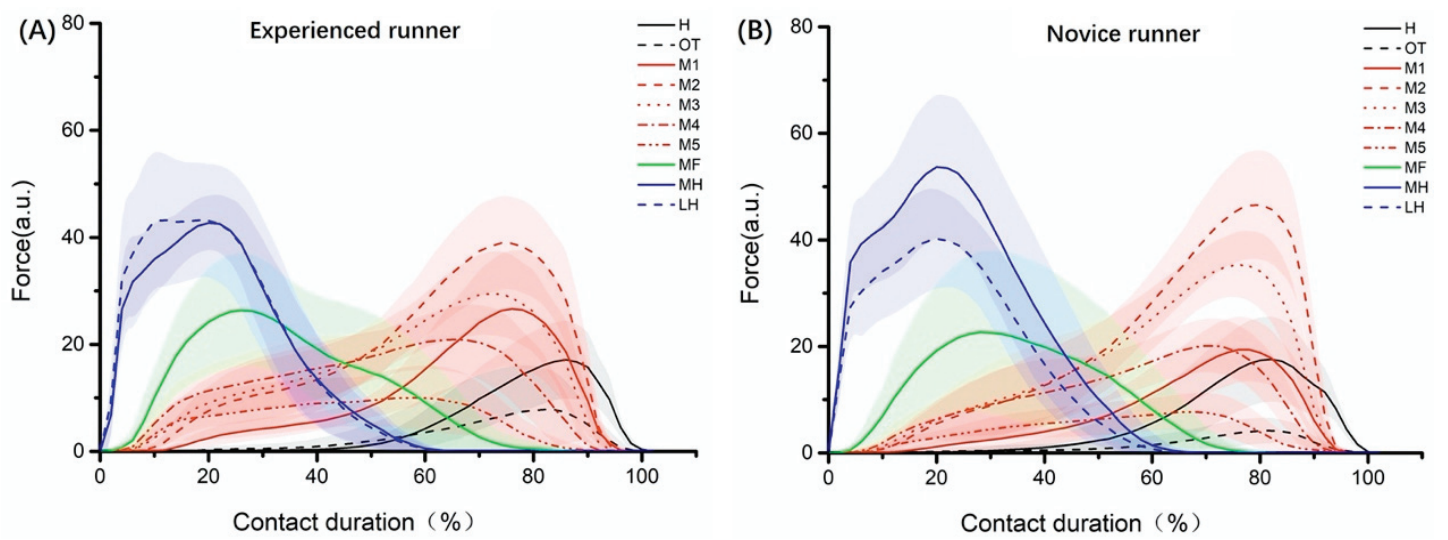

Fig. 1. The force time-series for the marathon group (A) and control group (B) in 10 anatomical regions 
Force development

The (SPM) statistics of time-series normalized force in ten regions and total force during stance was presented in the Figs. 1 and 2. The forces in the dif-

Force
Experienced runner
Novice runner

P-value

Experienced runner vs Novice runner
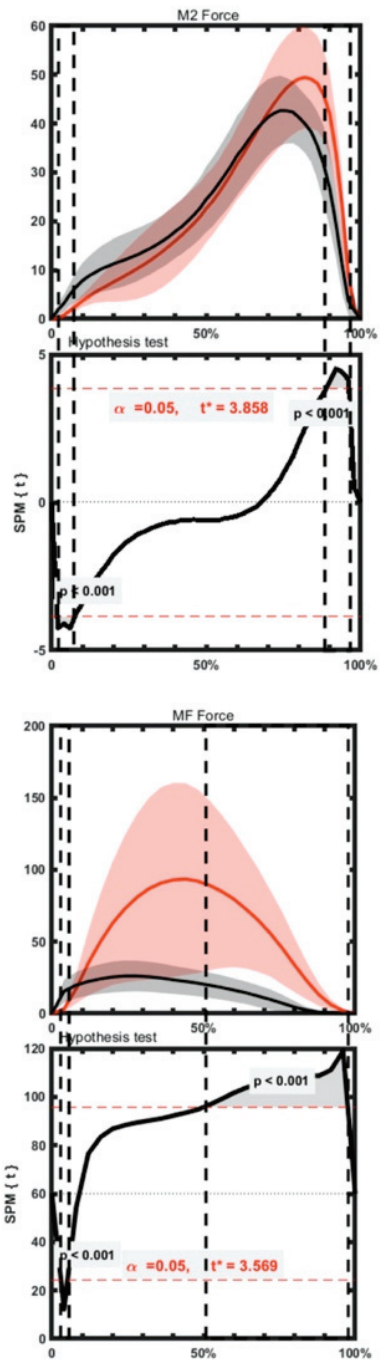
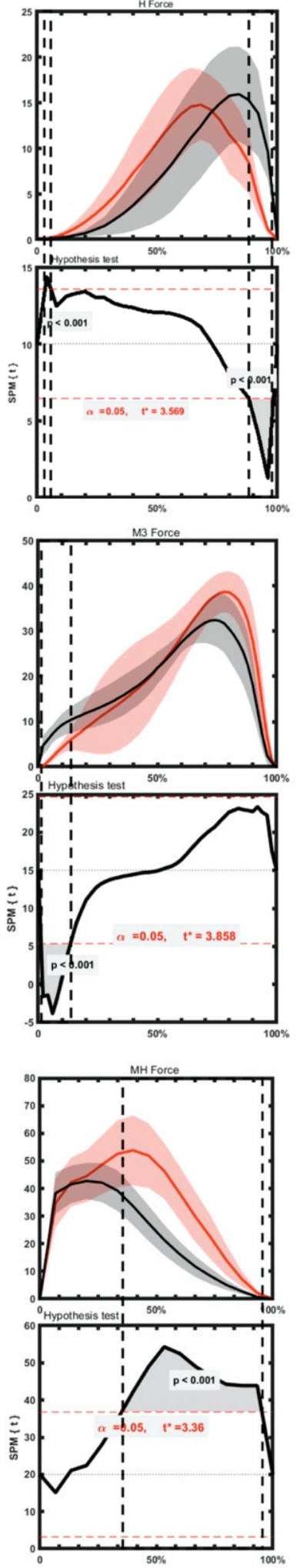

ferent regions between the experienced runners and the novice group differed throughout the stance. LH, $\mathrm{MH}, \mathrm{MF}, \mathrm{M} 5, \mathrm{M} 1$ and SUM showed the significant differences between the two groups $(p \leq 0.050)$. In $\mathrm{H}$ and OT, there were significant delays in occurring
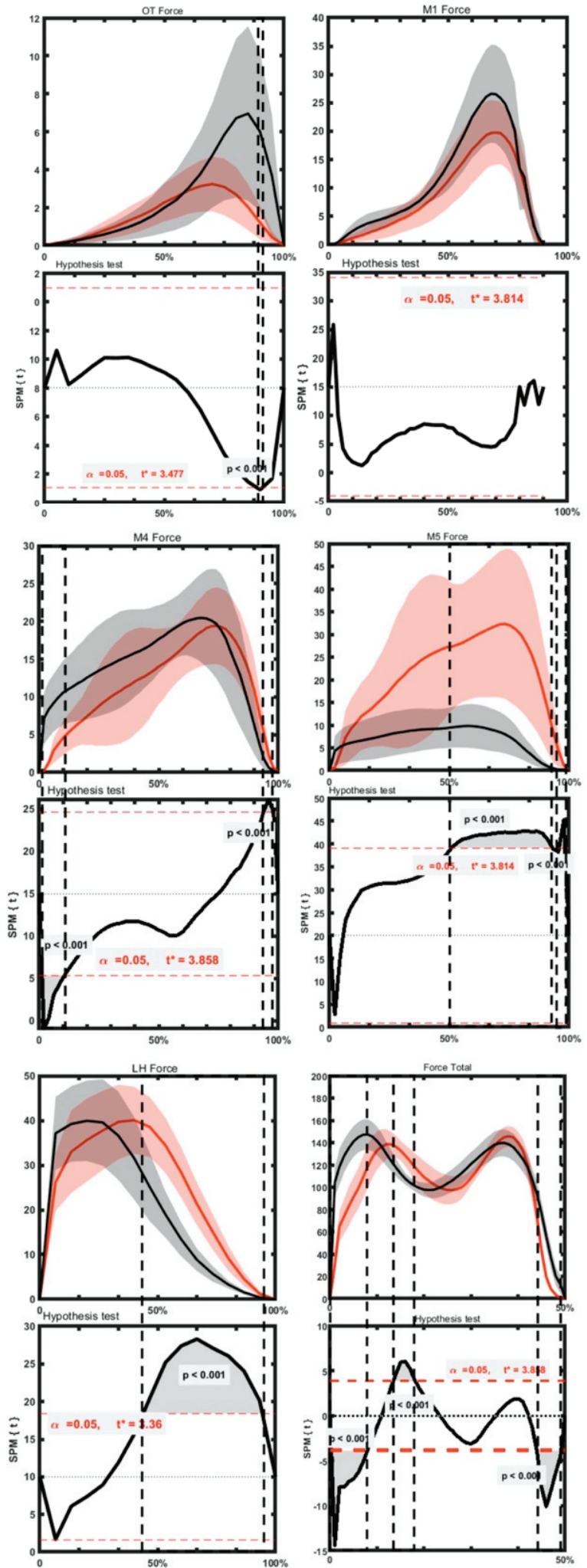

Fig. 2. Statistics from spm1d for time-series normalized force in 10 regions and total force between two groups 
Table 3. Time of peak force (\%) in Marathon group and Control group

\begin{tabular}{|l|c|c|c|c|c|}
\hline & Marathon group & Control group & $p$-value & Confidence interval (95\%) & Effect size \\
\hline H & $85.66(6.37)$ & $70.00(5.10)$ & $\mathbf{0 . 0 0 * *}$ & $(-20.50 \sim 10.83)$ & 0.81 \\
\hline OT & $83.55(2.37)$ & $71.11(6.84)$ & $\mathbf{0 . 0 0 * *}$ & $(-16.58 \sim-8.29)$ & 0.77 \\
\hline M1 & $76.48(2.42)$ & $76.67(5.23)$ & 0.91 & $(-3.46 \sim 3.83)$ & 0.02 \\
\hline M2 & $75.58(4.13)$ & $82.09(2.36)$ & $\mathbf{0 . 0 0 * *}$ & $(3.42 \sim 9.60)$ & 0.70 \\
\hline M3 & $72.19(7.56)$ & $77.39(10.15)$ & 0.18 & $(-2.60 \sim 12.99)$ & 0.28 \\
\hline M4 & $62.57(14.00)$ & $72.03(11.01)$ & 0.08 & $(-1.08 \sim 20.00)$ & 0.35 \\
\hline M5 & $58.50(12.83)$ & $64.49(16.63)$ & 0.35 & $(-6.91 \sim 18.90)$ & 0.20 \\
\hline MF & $31.82(7.17)$ & $46.92(11.68)$ & $\mathbf{0 . 0 0 * *}$ & $(6.56 \sim 23.65)$ & 0.61 \\
\hline MH & $25.00(7.05)$ & $41.25(8.16)$ & $\mathbf{0 . 0 0 * *}$ & $(9.67 \sim 22.83)$ & 0.73 \\
\hline LH & $21.59(8.58)$ & $37.92(8.68)$ & $\mathbf{0 . 0 0 * *}$ & $(9.96 \sim 23.69)$ & 0.69 \\
\hline Meta loading & $0.83(0.07)$ & $0.87(0.02)$ & $\mathbf{0 . 0 5 *}$ & $(0.01 \sim 0.10)$ & 0.44 \\
\hline Foot Balance & $0.89(0.03)$ & $0.76(0.30)$ & 0.11 & $(-0.31 \sim 0.04)$ & 0.31 \\
\hline
\end{tabular}

Note: “*” means significant difference between the two groups $(p \leq 0.05)$; “**” means significant difference between the two groups $(p \leq 0.01)$.

time of peak force, for the experienced runners ( $p \leq$ 0.001). In MF, MH, and $\mathrm{LH}$, significant delays in occurring of peak force were found in the novice group $(p \leq 0.001)$. The graph total of forces presented a typical walking pattern with passive (first) and active (second) peaks, with statistics presented in the Table 3. The Statistic Parametric Mapping (SPM) statistics of time-series normalized foot balance and meta loading parameters during stance was presented in the Fig. 3. The meta loading showed that the novice group was significantly larger than the experienced runners in peak meta loading ( $p \leq 0.001)$, and the occurring time of peak meta loading was significantly delayed comparing to experienced runners $(p=0.050)$. The peak foot balance was higher in novice group ( $p \leq 0.001)$, but no significance was observed in the occurring time of peak balance between two groups.

\section{Discussion}

This study assessed the differences of walking plantar pressures between the experienced runners and novice group to further explore the alterations of the foot functions from long-term prolonged running. Findings obtained in this study supported the hypothesis that significant differences in the plantar pressure, contact duration, contact area, medial-lateral (foot balance) loading and meta (central forefoot) loading, were found in the experienced runners during walking. Consistent with the previous report that altered movement control strategies may be developed with increased running experience, thus optimizing task performance [10], [25]. A further evidence of adjust- ing capability in the speed recovery of experienced elite runners concluded that experienced runners could recover to normal speed faster than novice runners after the interference of running speed [1].

The contact duration of phases during stance could illustrate the changes in the functional behavior in the foot [15]. Nagel et al. [21] found that contact time in the heel, midfoot and metatarsals increased significantly after a single marathon run. However, this study found the experienced runners presented reduced contact time in ICP, FFCP and FFPOP, compared to the novice group during walking. The discrepancy may due to the protocol or criteria selected for analysis, as this study separated the stance into four phases while the above-mentioned results came from times of each specific foot plantar regions. Furthermore, this may mitigate impact transient in the runners, leading to an alteration for repetitive landing impact [19]. In terms of the reduced time in FFCP and FFPOP, it may attribute to the active motion of toes, which functioned as pushing the body up and forward during locomotion, and practically sharing the load focused on the metatarsal bones [9]. Additionally, increased contact area in M1 of experienced runners may be another evidence [9].

A recent study reported that the fatigue of foot flexors and dorsiflexors after a long run would alter the distribution of foot pressure [7]. The peak pressure in the M2, M3 and M5 regions increased after a single marathon and increased peak force and impulse in M2, M3, M4 and MH were found [21]. Above findings were examples of deviations in plantar pressure after an immediate prolonged run [7]. During a long run, the cumulative loading in the plantar forefoot regions could lead to fatigue or even minor damage to the metatarsal bone [21]. Failure to achieve a dynamic 

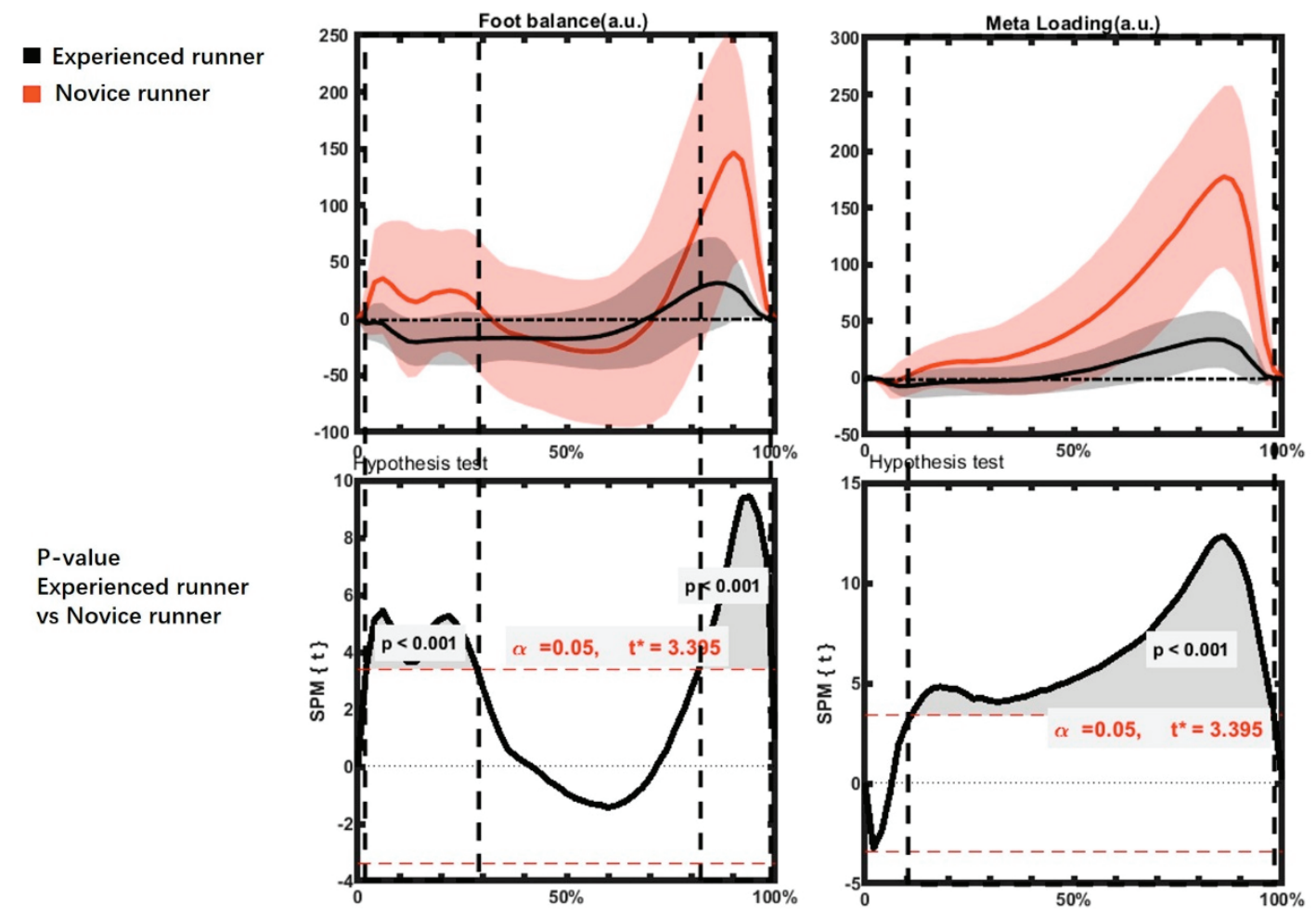

Fig. 3. Statistics from spm1d for (A) Meta loading and (B) Foot balance between two groups

balance between loading accumulation and bone remodeling may lead to pathological stress response, such as stress fracture [27]. The repetitive impact loading from long-distance running under metatarsal heads is a potential reason of metatarsal stress fractures [21]. However, as opposed to the above-mentioned literature, the findings of this study showed smaller peak pressure in M2 and maximal force in M3 of the experienced runners during walking. Another result of interesting and consolidating evidence was that the maximal force and impulse increased significantly in the OT of the experienced runners during walking, which could be explained with enhanced functional performance [14]. This change could, in turn, share load focalized in the metatarsal region from previously reported loading concentration after a single marathon run. As reported, the primary function of grasping and ambulation [14]. In this study, the increased maximal force and impulse in the OT of the experienced runners further indicated that grasping function obtained from long-term prolonged running, which was reported could be functioned as improving the performance of endurance running [24].

Agresta et al. [1] found no correlation between increased running experience and injury prevention. However, van Gent et al. [25] indicated that runners usually suffered from injuries to the feet, and running experience could effectively reduce the risk of injury. A recent study showed the increased pressure to the medial foot of the amateur runners after a long-distance run [6]. In this study, plantar loading of experienced runners was revealed, and the smaller maximal force, peak pressure and impulse in the heel region of the experienced runners could further support the aboveexplained reduced impact transient and soft heel strike. It may be a protective mechanism obtained from long-term prolonged running.

The comparison using 1-dimensional statistical parametric mapping provided approach to reveal message apart from extremum [22]. According to the timeseries force in each plantar region, the maximal force in $\mathrm{H}$ and $\mathrm{OT}$ regions were found with significantly delayed occurring time in the experienced runners during walking, which may adversely mean increased impulse to the $\mathrm{H}$ and OT thus assisting forefoot load sharing and pushing off. However, the maximal force in MF, MH and LH regions were significantly delayed in the novice group during walking, which may be due to the reduced impact transient in the experienced runners. What deserves special attention was the difference in the MF an M5. In this study, larger maximal force was found in the novice group, opposite to smaller force in the experienced runners during walking. However, as reported lateral forefoot (M5) was the region susceptible of stress fracture [21], the experienced runners in this study may adopt changes to offload this region. Additionally, arch drop from a single longdistance run with increased loading in the MF region 
[21], which was not the case of this study, may further consolidate the alteration in experienced runners, or possibly enhanced windlass mechanism [4]. Knowledge from this part may provide implication for athletic coaches, physical trainers, and physiotherapists that the practice of dynamic arch attenuation functions in the foot for prevention of overused injuries from repetitive load accumulation.

As presented in Fig. 3, the foot balance reflected the loading shifts in the medial or lateral foot during stance, and meta loading reflected the force distribution in the central metatarsals (M2 and M3) against M1 in the medial and M4 and M5 in the lateral sides. The significantly smaller variation of the foot balance in the experienced runners during initial contact and push off reveal that less medial-lateral plantar loading shifts, which might possibly indicate less pronationsupination motion in the experienced runners, as previously reported foot pronation from a single long distance run may be an indicator of increased knee and ankle loading [17]. Overloading in the metatarsals may lead to stress fracture from middle and long distance running, especially the impact loading in the central region of the forefoot, particularly, the second and third metatarsal bones [21]. The Meta loading showed that the peak value in the experienced runners was significantly smaller than the novice group and the time of peak force was shorter (less accumulative force, or impulses), which suggests less impact force or impulse concentrated to the second and third metatarsals. This may be due to a portion of the medial forefoot load being transferred to the OT region, thereby reducing the risk of M2 and M3 metatarsal injury. Our findings suggest that experienced runners may employed an adapted plantar loading distribution strategy to offload regions of M2 and M3, thus avoiding fatigue stress in the metatarsals, which are quite common among runners.

In conclusion, this study revealed the changes in the walking plantar pressures while comparing experienced runners against physical active novice runners. However, several limitations should be considered for this study that, only parameters in the plantar pressure were investigated, further studies should focus on more kinematic and kinetic variables from longitudinal study, which may provide additional information concerning adaption from long-term prolonged running. We utilized the statistical parametric mapping 1d package to reveal the difference in the timevarying force in each plantar region, however, further advanced statistical investigations of pressures mapping are suggested. Another point that plantar pressure measurement was conducted in the lab-based experi- ment, which might not be generable to the external real-life surroundings. Further studies with portable measurement system shall be taken for the analysis. Findings in this study highlighted the differences, specifically the contact duration of phases during stance, toes functions and medial-lateral loading shifting (foot balance) and metatarsals (central forefoot) offloading from long-term prolonged running.

\section{Acknowledgements}

This study was sponsored by the National Natural Science Foundation of China (No. 81772423), and K.C. Wong Magna Fund in Ningbo University.

\section{References}

[1] Agresta C.E., Peacock J., Housner J., Zernicke R.F., ZENDLER J.D., Experience does not influence injury-related joint kinematics and kinetics in distance runners, Gait and Posture, 2018, 61, 13-18, DOI: 10.1016/j.gaitpost.2017.12.020.

[2] Anbarian M., Esmaili H., The Effect of Running-induced Fatigue on Foot Roll-over Pattern in Novice Runners, Journal of Paramedical Sciences and Rehabilitation, 2016, 5 (4), 23-32.

[3] Bock W.J., The Definition and Recognition of Biological Adaptation, American Zoologist, 198020 (1), 217-227, DOI: 10.1093/icb/20.1.217.

[4] JiAng C., The Effect of Basketball Shoe Collar on Ankle Stability: A Systematic Review and Meta-Analysis, Physical Activity and Health, 2020, 4(1), 11-18, DOI: http://doi.org/ 10.5334/paah.48

[5] Decock A., Willems T., Witvrouw E., Vanrenterghem J., DECLERCQ D., A functional foot type classification with cluster analysis based on plantar pressure distribution during jogging, Gait and Posture, 2006, 23 (3), 339-347, DOI: 10.1016/j.gaitpost.2005.04.011.

[6] Hawrylak A., Matner P., Demidaś A., BarczyK-Pawelec K., DEMCZUK-WŁodARCZYK E., Static and dynamic plantar pressure distribution in amateur marathon runners, The Journal of Sports Medicine and Physical Fitness, 2019, 59 (1), 76-81, DOI: $10.23736 / \mathrm{s} 0022-4707.18 .07964-1$.

[7] HazzaA WalaA Eldin A., Mattes K., Influence of Foot Strike Pattern and Local Fatigue of Plantar Flexors and Dorsiflexors on Plantar Pressure during Running, German Journal of Sports Medicine, 2018, 69 (1), 19-25.

[8] Hessert M.J., Vyas M., Leach J., Hu K., Lipsitz L.A., NOVAK V., Foot pressure distribution during walking in young and old adults, BMC Geriatrics, 2005, 5 (1), 1-8, DOI: 10.1186/ 1471-2318-5-8.

[9] Hughes J., Clark P., Klenerman L., The importance of the toes in walking, Journal of Bone and Joint Surgery, 1990, 72 (2), 245-251, DOI: 10.1007/BF02470778.

[10] JOYNER M.J., COYLE E.F., Endurance exercise performance: the physiology of champions, The Journal of Physiology, 2008, 586 (1), 35-44, DOI: 10.1113/jphysiol.2007.143834.

[11] Kemler E., Blokland D., Backx F., Huisstede B., Differences in injury risk and characteristics of injuries between novice and experienced runners over a 4-year period, The 
Physician and Sportsmedicine, 2018, 46 (4), 485-491, DOI: 10.1080/00913847.2018.1507410.

[12] KluitenBerg B., MidDelKoOP M.V., Diercks R., WorP H.V.D., What are the Differences in Injury Proportions Between Different Populations of Runners? A Systematic Review and Meta-Analysis, Sports Medicine, 2015, 45 (8), 1143-1161, DOI: 10.1007/s40279-015-0331-x.

[13] Knechtle B., Scheer V., Nikolaidis P.T., Sousa C.V., Participation and Performance Trends in the Oldest 100-km Ultramarathon in the World, International Journal of Environmental Research and Public Health, 2020, 17 (5), 1-12, DOI: $10.3390 /$ ijerph17051719.

[14] Lambrinudi C., Use and Abuse of Toes, Postgraduate Medical Journal, 1932, 8 (86), 459-464, DOI: 10.1136/ pgmj.8.86.459.

[15] Mei Q., Gu Y., Fernandez J., Alterations of pregnant gait during pregnancy and post-partum, Scientific Reports, 2018, 8 (1), 1-7, DOI: 10.1038/s41598-018-20648-y.

[16] Mei Q., Fernandes J., Hume P., Gu Y., Investigating biomechanical function of toes through external manipulation integrating analysis, Acta Bioeng. Biomech., 2016, 18 (1), 97-102, DOI: 10.5277/ABB-00360-2015-02.

[17] Mei Q., Gu Y., Xiang L., BaKer J.S., Fernandez J., Foot pronation contributes to altered lower extremity loading after long distance running, Frontiers in Physiology, 2019, 10 (573), DOI: $10.3389 /$ fphys.2019.00573.

[18] Mei Q., Gu Y., Sun D., Fernandez J., How foot morphology changes influence shoe comfort and plantar pressure before and after long distance running?, Acta Bioeng. Biomech., 2018, 20 (2), 179-186, DOI: 10.5277/ABB-01112-2018-02.

[19] Morgan D.W., Martin P.E., Krahenbuhl G.S., Factors affecting running economy, Sports Med., 1989, 7 (5), 310-330.

[20] Mueller M.J., Application of plantar pressure assessment in footwear and insert design, Journal of Orthopaedic and Sports
Physical Therapy, 1999, 29 (12), 747-755, DOI: 10.2519/ jospt.1999.29.12.747.

[21] Nagel A., Fernholz F., Kibele C., Rosenbaum D., Long distance running increases plantar pressures beneath the metatarsal heads: a barefoot walking investigation of 200 marathon runners, Gait and Posture, 2008, 27 (1), 152-155, DOI: 10.1016/j.gaitpost.2006.12.012.

[22] PATAKy T.C., VANRENTERghem J., Robinson M.A., Two-way ANOVA for scalar trajectories, with experimental evidence of non-phasic interactions, Journal of Biomechanics, 2015, 48 (1), 186-189, DOI: 10.1016/j.jbiomech.2014.10.013.

[23] Razak A.H.A., Zayegh A., BegG R.K., Wahab Y., Foot plantar pressure measurement system: A review, Sensors, 2012, 12 (7), 9884-9912, DOI: 10.3390/s120709884.

[24] Rolian C., Lieberman D.E., Hamill J., Scott J.W., WERBEL W., Walking, running and the evolution of short toes in humans, Journal of Experimental Biology, 2009, 212 (5), 713-721, DOI: $10.1242 /$ jeb.019885.

[25] Van Gent R., Siem D., Van Middelkoop M., Van Os A.G., BIERMA-ZeInStRA S.M.A., KoEs B.W., Incidence and determinants of lower extremity running injuries in long distance runners: a systematic review, British Journal of Sports Medicine, 2007, 41(8), 469-480, DOI: 10.1136/bjsm.2006.033548.

[26] Vitez L., ZuPeT P., ZADNiK V., DrobNič M., Running injuries in the participants of Ljubljana Marathon, Slovenian Journal of Public Health, 2017, 56(4), 196-202, DOI: 10.1515/ sjph-2017-0027.

[27] Warden S.J., Burr D.B., Brukner P.D., Stress fractures: pathophysiology, epidemiology, and risk factors, Current Osteoporosis Reports, 2006, 4 (3), 103-109, DOI: 10.1007/s11914996-0029-y.

[28] Wen J., Ding Q., Yu Z., Sun W., Wang Q., WeI K., Adapt+ive changes of foot pressure in hallux valgus patients, Gait and Posture, 2012, 36 (3), 344-349, DOI: 10.1016/j.gaitpost.2012.03.030. 